\title{
Appendix I
}

Procedure for developing the artificial neural network (ANN) technique for Carboxyhaemoglobin-Potassium phosphate system.

The first step involved was data analysis and preprocessing. Temperature, $\mathrm{pH}$ and phosphate concentrations were chosen as input parameters. Carboxyhaemoglobin solubility decreases as temperature increases at high salt concentrations. Inverse of the temperature was therefore used as input parameter. Solubility falls exponentially as salt concentration is increased at a given $\mathrm{pH}$ and temperature. Hence logarithm of solubility was used as the dependent parameter (i.e. output). The inputs and outputs were then scaled to avoid numerical overflows during training. Since sigmoid (logistic) form of activation was used the input and output data for all the systems was scaled between 0.05 and 0.95 according to

$$
x_{i j}^{*}=a\left(x_{i j}-x_{\min }^{j}\right) /\left(x_{\max }^{j}-x_{\min }^{j}\right)+b
$$

where $x_{i j}^{*}$ and $x_{i j}$ refer to the normalized and unnormalized matrix elements (patterns). $x_{\text {max }}^{j}$ represents the element of $j^{t h}$ column having the largest magnitude and $x_{\text {min }}^{j}$ represents the element of $j^{\text {th }}$ column having the smallest magnitude. $a$ and $b$ are constants of the equation. This scaling strategy limits the scaled quantities in the range $[\mathrm{b},(\mathrm{a}+\mathrm{b})]$. The scaled data was then randomized.

\section{Training of neural network}

The carboxyhaemoglobin-potassium phosphate system consisting of 89 patterns was divided into 60 patterns for training, 15 patterns for validation and 14 patterns for testing. The ANN technique was carried out using the Stuttgart Neural Network simulator (SNNS) (version 4.2). The training, testing and validation pattern files were converted to format compatible with SNNS and loaded. A model consisting of 3 input neurons, 1 hidden neuron and 1 output neuron with full connectivity was constructed in SNNS. The weights are initialized to random values and then adjusted according to an error minimization technique until the error falls to an acceptable level. The activation function employed was logistic and the learning function was Standard backpropagation.

The training set, trains the network by minimizing the error between the predicted and experimental patterns. During the training after a defined number of cycles the model is used to calculate the error on the validation set. As the training progresses the training error decreases but after a certain number of cycles the validation error begins to increase. The training is stopped at this stage. Neural networks with 1 to 10 hidden layer neurons were trained to arrive at the optimum number of hidden layer neurons, $\mathrm{n}$. 